\title{
Co- and multimorbidity patterns in primary care based on episodes of care: results from the German CONTENT project Gunter Laux*, Thomas Kuehlein, Thomas Rosemann and Joachim Szecsenyi
}

Address: Department of General Practice and Health Services Research, University of Heidelberg, Germany

Email: Gunter Laux* - gunter.laux@med.uni-heidelberg.de; Thomas Kuehlein - thomas.kuehlein@med.uni-heidelberg.de; Thomas Rosemann - thomas.rosemann@med.uni-heidelberg.de; Joachim Szecsenyi - joachim.szecsenyi@med.uni-heidelberg.de

* Corresponding author

Published: 18 January 2008

BMC Health Services Research 2008, 8:14 doi:10.1 186/1472-6963-8-14
Received: 3 May 2007

Accepted: 18 January 2008

This article is available from: http://www.biomedcentral.com/1472-6963/8//4

(c) 2008 Laux et al; licensee BioMed Central Ltd.

This is an Open Access article distributed under the terms of the Creative Commons Attribution License (http://creativecommons.org/licenses/by/2.0), which permits unrestricted use, distribution, and reproduction in any medium, provided the original work is properly cited.

\begin{abstract}
Background: Due to technological progress and improvements in medical care and health policy the average age of patients in primary care is continuously growing. In equal measure, an increasing proportion of mostly elderly primary care patients presents with multiple coexisting medical conditions. To properly assess the current situation of co- and multimorbidity, valid scientific data based on an appropriate data structure are indispensable. CONTENT (CONTinuous morbidity registration Epidemiologic NeTwork) is an ambitious project in Germany to establish a system for adequate record keeping and analysis in primary care based on episodes of care. An episode is defined as health problem from its first presentation by a patient to a doctor until the completion of the last encounter for it. The study aims to describe co- and multimorbidity as well as health care utilization based on episodes of care for the study population of the first participating general practices.
\end{abstract}

Methods: The analyses were based on a total of 39,699 patients in a yearly contact group (YCG) out of 17 general practices in Germany for which data entry based on episodes of care using the International Classification of Primary Care (ICPC) was performed between I.I.2006 and 3I.12.2006. In order to model the relationship between the explanatory variables (age, gender, number of chronic conditions) and the response variables of interest (number of different prescriptions, number of referrals, number of encounters) that were applied to measure health care utilization, we used multiple linear regression.

Results: In comparison to gender, patients' age had a manifestly stronger impact on the number of different prescriptions, the number of referrals and number of encounters. In comparison to age $(\beta=0.043, p<0.000 \mathrm{I})$, multimorbidity measured by the number of patients' chronic conditions ( $\beta$ $=0.5 \mathrm{I}, \mathrm{p}<0.000 \mathrm{I})$ had a manifestly stronger impact the number of encounters for the observation period. Moreover, we could observe that the number of patients' chronic conditions had a significant impact on the number of different prescriptions $(\beta=0.226, p<0.000 \mathrm{I})$ as well as on the number of referrals $(\beta=0.3, p<0.000 \mathrm{I})$.

Conclusion: Documentation in primary care on the basis of episodes of care facilitates an insight to concurrently existing health problems and related medical procedures. Therefore, the resulting data provide a basis to obtain co- and multimorbidity patterns and corresponding health care utilization issues in order to understand the particular complex needs caused by multimorbidity. 


\section{Background}

Based upon technological progress and improvements in medical care and health policy, a growing number of patients survive medical conditions that used to be fatal formerly. As a result of this, an increasing proportion of mostly elderly primary care patients presents with multiple coexisting medical conditions. A large number of epidemiological studies from several countries support this estimate [1-4]. It could be shown that the risk of avoidable admissions and preventable complications increases dramatically with the number of chronic conditions [4]. Generally, it becomes more and more important to understand the particular complex needs caused by multimorbidity. However, to properly assess the current situation of multimorbidity and to draw recommendations for improvement, valid scientific data are indispensable.

In the German health care system, the GP (general practitioner) has some kind of gate-keeper role since patients who visit a specialist, without visiting the GP in advance have to pay an additional fee. In consequence, most visits to specialists are preceded by a GP consultation. The medical insurances cover all costs of routine care, including all visits to GPs as well as to specialists. Only some complementary and alternative medical treatments are not covered. A medical un-insurance does not exist, since a recently enacted law forces everyone to insure himself. In Germany everyone has free and unlimited access to the medical system and the vast majority of people uses the GP as entry into this system.

Electronic patient records in Germany are predominantly used for billing purposes. Thus, hitherto existing German routine data are unlikely to yield a realistic and differentiated picture of morbidity and health care utilization in primary care.

CONTENT (CONTinuous morbidity registration Epidemiologic NeTwork) is an ambitious project in Germany to establish a system for adequate record keeping and analysis in primary care. A scientific network was established consisting of participating surgeries, scientists, and statisticians. The aims are strictly scientific and the underlying hypothesis is that the knowledge-gaining process can be accelerated by combining the experience of many, especially with respect to complex interactions of factors and the analysis of rare events. The CONTENT EPR [5] (Electronic Patient Record) is based on the ICPC-2-R [6] (International Classification of Primary Care, $2^{\text {nd }}$ Revision) and allows a documentation in an episode of care structure over time. ICPC was accepted by the World Health Organization (WHO) as a related classification to be used for health information recording in primary care. The electronic version of the 2 nd ICPC edition (ICPC-2-E) is available for the use in electronic medical records [7].
Generally, there is a broad consensus that ICPC exactly meets the needs in primary care both in research as well as in practice and will add knowledge about morbidity patterns in this field.

An episode of care is defined as health problem from its first presentation by a patient to a doctor until the completion of the last encounter for it or presumably death, if the focal problem still exists [6]. An episode of care (in this study) actually includes all GP encounter elements none from specialists. Medical documentation in an episode of care character facilitates an insight to concurrently existing health problems and related medical procedures (e.g. prescriptions, referrals and hospitalization) and therefore provides a basis to obtain multimorbidity patterns. The CONTENT database has already yielded analyses that were impossible to achieve from German routine health care data [8].

We used the term multimorbidity to describe the cooccurrence of two or more chronic conditions as defined by van den Akker et al. [1]. The term comorbidity is used to describe the co-occurrence of medical conditions additional to an index disease as defined by Feinstein [9].

This study aims to describe co- and multimorbidity as well as health care utilization based on episodes of care, taking into account age and gender for the study population of the first 17 participating general practices.

\section{Methods}

A software module was developed to enable the coding of reasons for encounter, diagnoses and medical procedures with ICPC and assigning these issues to episodes of care. The module was integrated in an existing practice software to be used by voluntarily participating GPs. The extended practice software features a special function for data export based on XML (eXtensible Markup Language). The resulting data files are sent to the center in Heidelberg via email or upload to a dedicated server. In addition to the actual patient data the files contain meta data with information about the observation period and the surgery. To assure data quality the practices obtain feedback reports at regular intervals containing operating figures about episode based data entry with ICPC (e.g. the percentage of encounters without a documented reason for encounter). Moreover, these figures are discussed in periodic meetings with the GPs in order to continuously improve the data quality.

As a basic principle, only anonymized data are transmitted. For each patient, the CONTENT EPR contains a case number, the year of birth and the gender but not patients' names or addresses. Thus, it is not possible to determine a patient's identity and the implementation of extensive 
data security mechanisms is not needed. Moreover, the German Data Protection Act allows the transmission of anonymized patient data for scientific purposes without an explicit compliance of the patients. The study protocol was approved by the ethics committee of the University of Heidelberg (approval number 442/2005).

The data stem from a total of 39,699 patients in a Yearly Contact Group (YCG) out of 17 general practices with a total of 24 GPs located in 4 different federal states in West Germany with a concentration in Baden-Württemberg and Hessen. The YCG can be considered as an appropriate denominator since it is a good approximation of the "attending patients" in health systems with a patient list system $[10,11]$.

ICPC and episode based data entry was performed between 1.1.2006 and 31.12.2006. For these patients data about age, gender and episode based diagnoses were available as well as the corresponding medical procedures (different prescriptions and referrals). The number of different prescriptions per patient was determined at the $4^{\text {th }}$ level of the ATC (Anatomical Therapeutic Chemical Classification). The $4^{\text {th }}$ level determines the chemical or therapeutic or pharmacological subgroup. This is the level usually used to count "number of different drugs" as it is the level which aggregates drugs just above their descriptive chemical substance. The underlying referral list included all referrals to specialists (incl. repeat referrals) for the observed YCG.

On the basis of ICPC codes for the presented sample it was possible to define chronic conditions by using the concept of O'Halloran et al. that regards diagnoses as well as few chronic symptoms and complaints [12].

In order to model the relationship between the explanatory variables (age, gender, number of chronic conditions) and the response variables of interest (number of different prescriptions, number of referrals, number of encounters) we used multiple linear regression. Odds ratios were calculated by logistic regression. On account of the cluster sample study design the calculations were adjusted for the cluster (i.e. practice) on the basis of calculated ICCs (IntraCluster-Correlations). Statistical calculations were performed with SPSS version 14.0 and SAS version 8.0.

The study protocol was approved by the ethics committee of the University of Heidelberg (approval number 442/ 2005).

\section{Results}

For 39,699 patients in the YCG a total of 76,428 different episodes of care with an average of $1.87 \pm 0.02$ chronic conditions per patient were processed. $40.4 \%$ of the patients were male and $59.6 \%$ were female. The average age of the patients was $48.8 \pm 0.17$ years.

To get an impression of multimorbidity regarding age and gender we considered the average number of different chronic conditions per patient in a YCG. Moreover, we considered the number of different prescriptions as well as the number of referrals per patient stratified for age and gender (Table 1 and Figures $1 \mathrm{~A}$ to $1 \mathrm{C}$ ). The figures already point in the direction that patient's age appears to play an important role in context with the above mentioned variables for both male and female patients.

To examine more precisely the dependence of the response variables (number of different prescriptions, number of referrals, number of encounters) on age, gender and number of chronic conditions we performed a multiple linear regression. Table 2 shows the corresponding results.

Generally, in comparison to gender, the influence of age on the response variables was notably stronger, displayed by the regression coefficient $\beta$, that was standardized for the particular range. It could be observed that the impact of gender on the number of referrals $(\beta=0.053, \mathrm{p}<$ $0.0001)$ was predominantly associated with referrals of women to gynaecologists.

In comparison to age $(\beta=0.043, \mathrm{p}<0.0001)$, multimorbidity had a manifestly stronger impact on the number of encounters $(\beta=0.51, \mathrm{p}<0.0001)$ within our regression model. Moreover, we could observe that the number of patients' chronic conditions had a significant impact on the number of different prescriptions $(\beta=0.226, \mathrm{p}<$ $0.0001)$ as well as on the number of referrals $(\beta=0.3, p<$ $0.0001)$.

The CONTENT database also facilitates to describe the cooccurrence of medical conditions additional to an index disease. We wanted to describe the prevalence and the extent of comorbidities for the following highly prevalent chronic diseases: hypertension (K86/87), chronic ischemic heart disease (K74-76), diabetes mellitus (T89/ 90), and osteoarthrosis (L89-91). It has to be regarded that the prevalence estimates are based on the entry of the selected problem within the YCG in the observation period and do not refer to patients' lifetime. Table 3 shows that these chronic diseases feature a strong cohesion both for male and female patients. Moreover, the highly prevalent diseases lipid disorder (T93) and back syndrome (L84) were associated with these diseases. 
A

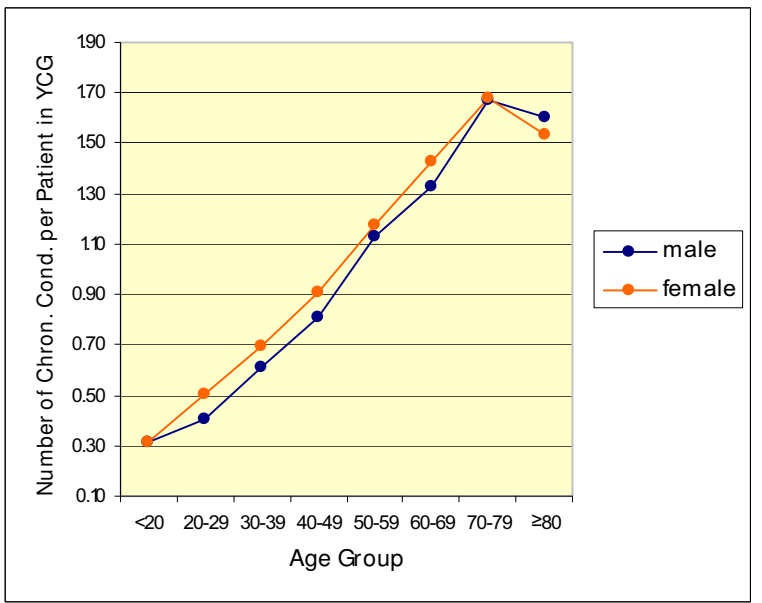

B

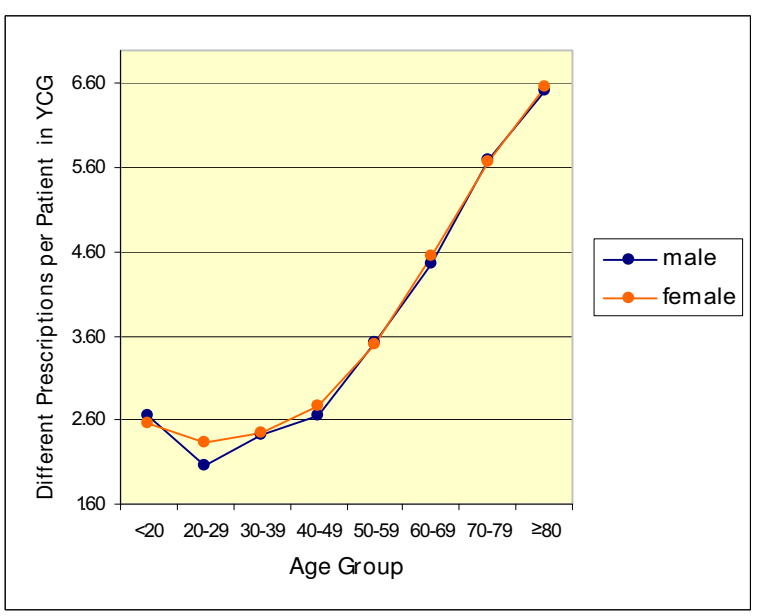

C

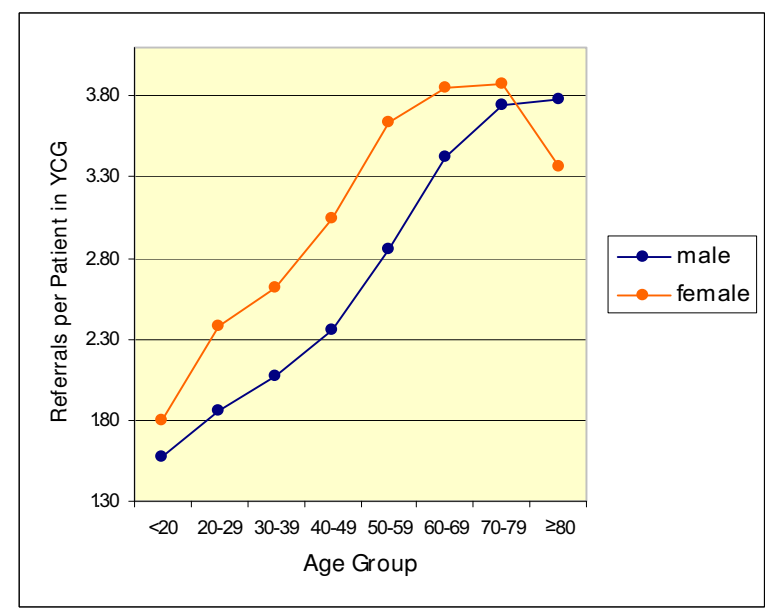

\section{Figure I}

Characteristics of multimorbidity and health care utilization. A - Average number of different chronic conditions per patient in YCG (yearly contact group). B - Average number of different prescriptions per patient in YCG. $C-A v e r a g e$ number of referrals per patient in YCG. 
Table I: Characteristics of multimorbidity and health care utilization

\begin{tabular}{|c|c|c|c|c|c|}
\hline Age Group (Years) & Gender & Patients (\%) & $\begin{array}{l}\text { Average number of } \\
\text { chronic conditions per } \\
\text { patient in YCG ( } \pm \text { SE) }\end{array}$ & $\begin{array}{c}\text { Average number of } \\
\text { different prescriptions } \\
\text { per patient in YCG ( } \pm \text { SE) }\end{array}$ & $\begin{array}{l}\text { Average number of } \\
\text { referrals per patient in } \\
\text { YCG }( \pm \mathrm{SE})\end{array}$ \\
\hline \multirow[t]{2}{*}{$<20$} & male & $2316(4 \mid .1)$ & $0.32 \pm 0.19$ & $2.64 \pm 0.21$ & $1.57 \pm 0.11$ \\
\hline & female & 3324 (58.9) & $0.32 \pm 0.18$ & $2.56 \pm 0.12$ & $1.80 \pm 0.12$ \\
\hline \multirow[t]{2}{*}{$20-29$} & male & $1847(37.9)$ & $0.41 \pm 0.21$ & $2.05 \pm 0.27$ & $1.85 \pm 0.13$ \\
\hline & female & $3028(62.1)$ & $0.50 \pm 0.19$ & $2.33 \pm 0.15$ & $2.38 \pm 0.11$ \\
\hline \multirow[t]{2}{*}{$30-39$} & male & $2110(38.9)$ & $0.61 \pm 0.21$ & $2.43 \pm 0.23$ & $2.07 \pm 0.14$ \\
\hline & female & $3322(61.1)$ & $0.69 \pm 0.18$ & $2.45 \pm 0.12$ & $2.62 \pm 0.09$ \\
\hline \multirow[t]{2}{*}{$40-49$} & male & $2868(41.5)$ & $0.81 \pm 0.17$ & $2.64 \pm 0.19$ & $2.35 \pm 0.12$ \\
\hline & female & $405 I(58.5)$ & $0.91 \pm 0.15$ & $2.77 \pm 0.13$ & $3.04 \pm 0.05$ \\
\hline \multirow[t]{2}{*}{ 50-59 } & male & $2324(44.3)$ & $1.13 \pm 0.18$ & $3.52 \pm 0.19$ & $2.85 \pm 0.11$ \\
\hline & female & 2919 (55.7) & $1.18 \pm 0.17$ & $3.50 \pm 0.15$ & $3.64 \pm 0.12$ \\
\hline \multirow[t]{2}{*}{$60-69$} & male & 2279 (44.9) & $1.33 \pm 0.19$ & $4.45 \pm 0.19$ & $3.43 \pm 0.11$ \\
\hline & female & $2791(55.1)$ & $1.43 \pm 0.16$ & $4.55 \pm 0.16$ & $3.85 \pm 0.09$ \\
\hline \multirow[t]{2}{*}{ 70-79 } & male & $1568(42.0)$ & $1.67 \pm 0.22$ & $5.70 \pm 0.31$ & $3.75 \pm 0.18$ \\
\hline & female & $2161(58.0)$ & $1.68 \pm 0.19$ & $5.67 \pm 0.22$ & $3.88 \pm 0.12$ \\
\hline \multirow[t]{2}{*}{$\geq \mathbf{8 0}$} & male & $737(25.3)$ & $1.60 \pm 0.43$ & $6.51 \pm 0.69$ & $3.78 \pm 0.40$ \\
\hline & female & $2174(74.7)$ & $1.53 \pm 0.21$ & $6.57 \pm 0.24$ & $3.36 \pm 0.15$ \\
\hline
\end{tabular}

\section{Discussion}

As an answer to our research question we found a strong correlation between age, gender, multimorbidity and health care utilization. These findings are not surprising and do not stand in contrast to comparable findings in the international scientific literature [1-4,13]. Nonetheless, our study is the first approach to this phenomenon in our country with an international classification developed for primary care.

Generally, when addressing multimorbidity issues in order to compare the results of different studies, possible differences concerning the research question, the data sources and the definition of multimorbidity have to be taken into account. Fortin et al. collected prevalence estimations of multimorbidity in Europe, the Middle East, the United States, and Canada. Since research questions, information collection, and multimorbidity measures differed, major differences in the results were observed [14]. However, there is a broad consensus that multimorbidity and its high prevalence is an important issue in family practice that deserves more scientific research [15].

Van den Aker et al. concluded that multimorbidity, although it increases with age, is a frequent phenomenon among all ages [1]. This phenomenon was also observed within our study sample. $12.8 \%$ of the patients younger than 50 years featured 2 or more chronic conditions. Therefore, research into multimorbidity should not only focus on the elderly, who are especially at risk.

Multimorbidity as defined by routinely collected data in electronic patient records not only offers an epidemiological overview of morbidity patterns for the scientist, but can also help the GP to identify patients with an increased likelihood of needing more attention [16]. We observed a typical clustering of specific health problems (e.g. diabetes, hypercholesterinemia and hypertension, Table 3). These clusters can be easily identified by the GP on the basis of the EPR in order to apply an appropriate medical

Table 2: Multiple linear regression analysis

\begin{tabular}{|c|c|c|c|c|}
\hline Response variable & Explanatory variables & $\begin{array}{r}\text { Proportion of explained } \\
\text { variance (adjusted } \mathbf{R}^{2} \text { ) }\end{array}$ & Adjusted regression coefficient $\beta$ & $\mathbf{p}$ \\
\hline \multirow[t]{3}{*}{ Different prescriptions } & Age & 0.28 & 0.315 & $<0.0001$ \\
\hline & Gender & & 0.008 & n.s. \\
\hline & Number of chronic conditions & & 0.226 & $<0.0001$ \\
\hline \multirow[t]{3}{*}{ Referrals } & Age & 0.39 & 0.178 & $<0.0001$ \\
\hline & Gender & & 0.053 & $<0.0001$ \\
\hline & Number of chronic conditions & & 0.300 & $<0.0001$ \\
\hline \multirow[t]{3}{*}{ Encounters } & Age & 0.37 & 0.043 & $<0.0001$ \\
\hline & Gender & & 0.029 & $<0.0001$ \\
\hline & Number of chronic conditions & & 0.510 & $<0.0001$ \\
\hline
\end{tabular}




\begin{tabular}{|c|c|c|c|c|c|c|c|c|}
\hline K86/87 Hypertension & 2385 & $1033(43.3)$ & $1352(56.7)$ & 60.01 & 64.41 & 57.14 & $\begin{array}{l}\text { T93 Lipid Disorder 20I }(19.5,8.4) \\
\text { K74-76 Chron.isc.heart d. I87(18.I, } \\
\text { 10.9) }\end{array}$ & $\begin{array}{l}\text { T93 Lipid Disorder 224(16.6, 8.I) } \\
\text { L89-L9I Osteoarthrosis } 222(16.4,7.8) \\
\text { T89/90 Diabetes mellitus } 221(16.3,10.2)\end{array}$ \\
\hline
\end{tabular}

84 Back syndrome 14

T99 Endocrine, other 79(7.6, 12.2$)$

K74-76 Chron.isc.heart d. I76(I3.0,

L89-L9I Osteoarthrosis $75(7.3,3.6)$ 14.0)

P76 Depressive Disorder II9(8.8, 3.5)

$\begin{array}{lllllll}\begin{array}{l}\text { K74-76 Chronic ischemic } \\ \text { heart disease }\end{array} & 955 & 507(53.1) & 448(46.9) & 24.06 & 31.61 & 19.93\end{array}$

K86/K87 Hypertension 159(31.4, 8.1)

T93 Lipid Disorder $121(23.9,9.5)$

T89/90 Diabetes mellitus $93(18.3,7.5)$

L84 Back syndrome 92(I8.I, I.9)

L89-L9I Osteoarthrosis $65(12.8,6.8)$

K80 Cardiac arrhythmia $49(9.7,9.9)$

K86/K87 Hypertension I7I(38.I, I2.5)

L89-L9I Osteoarthrosis 97(2I.7, 9.3)

T89/90 Diabetes mellitus 90(20.1, 10.4)

L84 Back syndrome 87 $(19.4,2.0)$

T93 Lipid Disorder 8I (I8.1, 7.4)

K77 Ischemic heart d. $71(I 5.8,23.4)$

\begin{tabular}{|c|c|c|c|c|c|c|c|c|}
\hline T89/90 Diabetes mellitus & 1225 & $566(45.1)$ & $659(54.9)$ & 31.61 & 35.29 & 27.85 & $\begin{array}{l}\text { K86/K87 Hypertension I52 }(26.9,6.4) \\
\text { T93 Lipid Disorder I0I }(17.8,6.3) \\
\text { K74-76 Chron.isc.heart d. } 99(17.5,8.3) \\
\text { L84 Back syndrome } 70(12.4,1.2) \\
\text { L89-L9I Osteoarthrosis } 61(10.8,5.5) \\
\text { T99 Endocrine, other } 41(7.2,8.6)\end{array}$ & $\begin{array}{l}\text { K86/K87 Hypertension 207(3I.4, 9.5) } \\
\text { T93 Lipid Disorder 99(I5.0, 6.0) } \\
\text { L89-L9I Osteoarthrosis } 98(\mid 4.9,5.8) \\
\text { K74-76 Chron.isc.heart d. } 90(13.6,1 \mid .2) \\
\text { L84 Back syndrome } 88(\mid 3.4,1.3) \\
\text { U99 Urinary disease, other } 71(10.8,2.7)\end{array}$ \\
\hline L89-9 I Osteoarthrosis & 1223 & 449 (36.7) & $774(63.3)$ & 30.81 & 28.0 & 32.71 & $\begin{array}{l}\text { L84 Back syndrome I0I }(22.5,2.6) \\
\text { K74-76 Chron.isc.heart d. 7I }(I 5.8,6.9) \\
\text { K86/K87 Hypertension } 69(15.3,2.9) \\
\text { T93 Lipid Disorder } 65(14.5,4.6) \\
\text { T89/90 Diabetes mellitus } 54(I 2.0,4.2) \\
\text { Y85 Benign prost. hyp. } 50(I I . I, 7.2)\end{array}$ & $\begin{array}{l}\text { L84 Back syndrome } 207(26.7,3.2) \\
\text { K86/K87 Hypertension } 202(26.1,7.2) \\
\text { T93 Lipid Disorder II I }(14.3,5.8) \\
\text { K74-76 Chron.isc.heart d. } 93(12.0,9.7) \\
\text { T89/90 Diabetes mellitus } 90(11.6,5.4) \\
\text { U99 Urinary disease, other } 84(10.8,2.7)\end{array}$ \\
\hline
\end{tabular}


care and to initiate specific interventions (e.g. lifestyle modifications).

\section{Limitations}

Generally, a potential selection bias must be admitted since the GPs' participation is voluntary and by now mainly focuses on Southwest Germany. Moreover, the number of 17 practices is still too small to draw strong conclusions.

In order to assess morbidity, there are several detailed and validated morbidity indexes [17]. For example, the "Cumulative Illness Rating Scale" (CIRS) [18] index additionally regards the severity of each condition and was also validated for the use to quantify multimorbidity for primary care patients [19]. However, since we had no information of the condition severity within the CONTENT EPR, we could not calculate this index for our study and had to limit on disease counts. Moreover, it would have been challenging to analyse the influence of sociodemografic factors (e.g. education, profession, income) on multimorbidity. However, sociodemografic information was only available for a small fraction of the sample.

The definition of a specific chronic condition on the basis of ICPC codes is often ambiguous. For example, we defined Osteoarthrosis (OA) by inclusion of ICPC codes L89 (Osteoarthrosis of hip), L90 (Osteoarthrosis of knee) and L91 (Osteoarthrosis, other). L84 (Back syndrome without radiating pain) is not included in our selection but also includes OA of the back. However, L84 also includes diseases that are not related to OA (e.g. back strain). Moreover, L91 includes 'arthritis unspecified' and 'traumatic arthropathy' that are not directly related to OA. This general problem could be solved by using a more specific terminology level which would allow grouping of all osteoarthrosis (no matter the site) from all applicable ICPC-2 codes.

\section{Strengths}

As mentioned above, the CONTENT project is the first approach in Germany based on episodes of care and ICPC that facilitates detailed long term analyses of co- and multimorbidity.

Especially, the continuous registration of patients' presented symptoms is new in comparison to hitherto existing German EPRs. Thus, CONTENT data enable to analyse the correlation between presented symptoms and resulting diagnoses in consideration of existing comorbidities. Moreover, age, gender as well as seasonal and regional differences have to be taken into account. In the long run, for every ICPC symptom (SY) it will be possible to determine a list $L$ of resulting diagnoses $D_{1}, \ldots ., D_{n}$ and corresponding probabilities $P_{1}, \ldots . ., P_{n}$ taking into account the above mentioned constraints (A: age, G: gender, $S$ : season, R: region, $C_{1}, \ldots . ., C_{m}$ : existing comorbidities), as the following formal description shows:

$$
L\left(S Y ; A, G, S, R, \sum_{i=1}^{m} C_{i}\right)=\left\{\begin{array}{ll}
D_{1}: & P_{1} \\
D_{2}: & P_{2} \\
& \\
& \\
D_{n}: & P_{n}
\end{array} \text { whereas } \sum_{j=1}^{n} P_{j}=1\right.
$$

This detailed model represents an extension of the model presented by Lamberts et al. [20].

\section{Conclusion}

We could observe a strong correlation between age, gender, multimorbidity and health care utilization in our study sample. Generally, documentation in primary care on the basis of episodes of care facilitates an insight to concurrently existing health problems and related medical procedures. Therefore, the resulting data provide a basis to obtain multimorbidity patterns and corresponding health care utilization issues. The continuously growing number of patients and practices has the potential to facilitate detailed long term analyses of co- and multimorbidity.

The increasing application of ICPC- and episode-based EPRs all over the world will allow challenging international comparisons in order to see national differences and regional distinctions and to discover what is generic in family practice and independent from local or national conditions. Further analyses will subsequently be based on the continuously expanding database and have the potential to shed light on complex epidemiological and health economics research questions.

\section{Competing interests}

The author(s) declare that they have no competing interests.

\section{Authors' contributions}

GL conceived and designed the study, organized and led data collection, analyzed and interpreted the data, and drafted the manuscript. TK assisted in data interpretation. TR assisted in study design and critically reviewed the manuscript. JS conceived and potentiated the superordinated project CONTENT. All authors read and approved the final manuscript.

\section{Acknowledgements}

The cooperation of the participating family doctors is greatly acknowledged. The superordinated project CONTENT was financed by the German Ministry of Education and Research (Bundesministerium für Bildung und Forschung, BMBF, grant number OIGK030I). 


\section{References}

I. vd Akker M, Buntinx F, Metsemakers JF, Roos S, Knottnerus JA: Multimorbidity in general practice: prevalence, incidence, and determinants of co-occurring chronic and recurrent diseases. I Clin Epidemiol 1998, 5 I:367-375.

2. Fortin M, Lapointe L, Hudon C, Vanasse A, Ntetu AL, Maltais D: Multimorbidity and quality of life in primary care: a systematic review. Health Qual Life Outcomes 2:5I. 2004 Sep 20;

3. Schellevis FG, vd Velden J, vd Lisdonk E, v Eijk JThM, v Weel C: Comorbidity of chronic diseases in general practice. J Clin Epidemiol 1993, 46:469-473.

4. Wolff JL, Starfield B, Anderson G: Prevalence, expenditures, and complications of multiple chronic conditions in elderly. Arch Intern Med 2002, I 62:2269-2276.

5. Laux G, Koerner T, Rosemann T, Beyer M, Gilbert K, Szecsenyi J: The CONTENT project: a problem-oriented, episode-based electronic patient record in primary care. Inform Prim Care 2005, I 3:249-255.

6. ICPC-2-R: International Classification of Primary Care. Revised Second Edition. Oxford: Oxford University Press; 2005.

7. Okkes IM, Jamoulle M, Lamberts H, Bentzen N: ICPC-2-E. The electronic version of ICPC-2. Differences with the printed version and the consequences. Fam Pract 2000, I 7:101-106.

8. Laux G, Rosemann T, Körner T, Heiderhoff M, Schneider A, Kühlein $\mathrm{T}$, Szecsenyi J: Detailed data collection regarding the utilization of medical services, morbidity, course of illness and outcomes by episode-based documentation in general practices within the CONTENT project. Gesundheitswesen 2007, 69(5):284-9l.

9. Feinstein A: The pre-therapeutic classification of co-morbidity in chronic disease. J Chron Dis 1970, 23:455-68.

10. De Loof J: Practice size. A fraction of the yearly attending group as practice size indicator. Allg Med Int 1983, I 2: I 27-I 28.

II. Krogh-Jensen P: Estimation of the practice population. The denominator in health systems with fee for services. Allg Med Int 1983, I 2:129-34.

12. O'Halloran J, Miller GC, Britt H: Defining chronic conditions for primary care with ICPC-2. Fam Pract 2004, 2 I :38 I-86.

13. Schellevis FG, Van de Lisdonk EH, Van der Velden J, Hoogbergen SH, Van Eijk JT, Van Weel C: Consultation rates and incidence of intercurrent morbidity among patients with chronic disease in general practice. Br J Gen Pract 1994, 44:259-262.

14. Fortin M, Bravo G, Hudon C, Vanasse A, Lapointe L: Prevalence of multimorbidity among adults seen in family practice. Ann Fam Med 2005, 3:223-8.

I5. Fortin M, Lapointe L, Hudon C, Vanasse A: Multimorbidity is common to family practice: is it commonly researched? Can Fam Physician 2005, 5 I:244-5.

16. Kadam U, Croft P: Clinical multimorbidity and physical function in older adults: a record and health status linkage study in general practice. Fam Pract 2007 in press.

17. de Groot V, Beckerman H, Lankhorst GJ, Bouter LM: How to measure comorbidity. a critical review of available methods. J Clin Epidemiol 2003, 56:221-29.

18. Linn BS, Linn MW, Gurel L: Cumulative illness rating scale. J Am Geriatr Soc 1968, 16:622-626.

19. Hudon C, Fortin M, Vanasse A: Cumulative Illness Rating Scale was a reliable and valid index in a family practice context. J Clin Epidemiol 2005, 58:603-608.

20. Lamberts H, Oskam SK, Okkes IM: The clinical relationship between symptoms and the final diagnosis in general practice, determined by means of posterior probabilities calculated on the basis of the Transition Project. Ned Tijdschr Geneeskd 2005, I49:2566-72.

\section{Pre-publication history}

The pre-publication history for this paper can be accessed here:

http://www.biomedcentral.com/1472-6963/8/14/prepub
Publish with Biomed Central and every scientist can read your work free of charge

"BioMed Central will be the most significant development for disseminating the results of biomedical research in our lifetime. "

Sir Paul Nurse, Cancer Research UK

Your research papers will be:

- available free of charge to the entire biomedical community

- peer reviewed and published immediately upon acceptance

- cited in PubMed and archived on PubMed Central

- yours - you keep the copyright

Submit your manuscript here:

http://www.biomedcentral.com/info/publishing_adv.asp
BioMedcentral 